\title{
2. Organisation des Tages und Teamarbeit
}

\author{
Alexander Knoke
}

Die Stationsarbeit ist die zentrale Aufgabe eines Arztes im Krankenhaus. Dennoch berichten viele Berufsanfänger, sich nur unzureichend auf den Stationsalltag vorbereitet zu fühlen. Denken Sie einen Moment nach: Wurde Ihnen im Studium vermittelt, wie es sich anfühlt, plötzlich zentrale Anlaufstelle für unterschiedlichste Berufsgruppen, Patienten und Angehörige zu sein? Hat irgendein Dozent Ihnen beigebracht, wie man verhindert, dass die Arztbriefe auf Ihrem Schreibtisch sich zu Türmen stapeln? Und was hatte Ihr Anatomieprofessor nochmal zum Thema Teamarbeit zu sagen? Herzlich wenig? Keine Panik, dann geht es Ihnen so wie allen anderen Berufsanfängern. Im ersten Teil dieses Kapitels geht es deshalb um die Organisation der Stationsarbeit, mit einem besonderen Augenmerk auf psychiatrietypische Aspekte. Im zweiten Teil geht es dann um die Teamarbeit, ohne die im Krankenhaus, und besonders in der Psychiatrie, gar nichts geht. Alles ist aus der eigenen Sichtweise, nämlich der eines Arztes in der Psychiatrie geschrieben. Falls Sie einer anderen medizinischen Fachrichtung angehören, so soll Sie dies aber bitte nicht davon abhalten, in diesem Kapitel trotzdem weiterzulesen. Wenn Sie einiges dabei in Ihr Fachgebiet übersetzen, wird es für Sie eine ebenso große Hilfe darstellen wie für die psychiatrischen Kollegen.

\section{Organisation des Tages}

\section{Was mache ich hier eigentlich?}

Neu auf Station - Was nun? Machen Sie sich erst einmal klar, was Ihre Aufgaben sind.

Da sind zunächst die festen Termine: Visiten, Teamsitzungen und Übergaben, Supervisionen, Fortbildungen, Gruppentherapien - es wird viel geredet und diskutiert in der Psychiatrie. Und Sie als Arzt sind gleich mittendrin. Sie werden sich plötzlich in einer Teamsitzung wiederfinden, wo alle darauf warten, dass Sie 
als Arzt Ihre Meinung äußern, dirigieren, vermitteln. Ein Angehöriger wird vielleicht gleichzeitig vor der Tür stehen und von Ihnen hören wollen, ob, wie und wann seine Schwester, Mutter, Tochter wieder gesund wird. Ein Patient steigert sich vielleicht gerade in einen Erregungszustand hinein, während ein anderer seine sofortige Entlassung wünscht. Und immer werden Sie gerufen. Deshalb hilft es, sich zwischen all der Plackerei mal zu fragen: Was wird eigentlich von Ihnen erwartet? Was erwartet die Pflege, was Ihre Kollegen, was Ihre Vorgesetzten, was die Patienten und Angehörigen von Ihnen? Und noch viel wichtiger: Was erwarten Sie von sich selbst? Was macht für Sie einen guten Arzt aus? Wie möchten Sie sein? Was müssen Sie am Tag schaffen, damit Sie zufrieden damit sind? In der Mühle Alltag vergisst man manchmal seine Prinzipien und Prioritäten. Ist dieses Antragsformular wirklich wichtiger als jener verzweifelte Angehörige? Oder muss es im Gegenzug sein, dass jener aufdringliche Patient Sie zum zehnten Mal am Tag davon abhält, einen wichtigen Arztbrief zu schreiben? Müssen immer Sie es sein, der in der Teamsitzung die Wortführung übernimmt oder kennt sich vielleicht jemand anderes in einem bestimmten Thema viel besser aus und wartet nur darauf, einbezogen zu werden? Fragen wie diese sollten Sie sich von Zeit zu Zeit stellen, um die Kontrolle über Ihr eigenes Hamsterrad zu behalten.

\section{Kleine Helfer}

Sie werden vielleicht schon mitbekommen haben, dass man im Stationsalltag durchaus mal den Überblick verlieren kann. Um dies zu verhindern, sollten Sie sich folgende kleine Helfer zunutze machen:

Zum einen hilft es, einen Kalender zu führen, sei er aus Papier oder digital. Spätestens, wenn Sie den ersten Termin mit einem gesetzlichen Betreuer vergessen haben und diesen hektisch in Ihren ohnehin vollen Tagesplan integrieren müssen, werden Sie an diesen Tipp denken.

Unverzichtbar ist außerdem ein kleines Notizbuch mit den wichtigsten Ansprechpartnern und Telefonnummern. Da hilft es meist, einen erfahrenen Kollegen zu fragen, um diese Nummern zu sammeln. Der Rest ergibt sich dann im Alltag. Spätestens nach dem ersten Anruf beim sozialpsychiatrischen Dienst oder beim zuständigen Gericht hat man auch diese Nummern. Notfallnummern, zu denen das Rea-Team, der Wachschutz, der Giftnotruf und die Polizei gehören, sollten vom ersten Tag an auf Seite eins stehen. Es gibt wenig Zeitraubenderes und Ärgerlicheres als das Suchen einer Telefonnummer in einer ohnehin bereits hektischen Situation.

Auch To-Do-Listen haben sich bewährt bzw. Patientenlisten mit einer „To-DoSpalte“. Hierbei ist jedoch darauf zu achten, dass man die Listen regelmäßig abarbeitet und entsorgt. Sonst hat man statt der erwarteten Ordnung ganz schnell für Zettelchaos und Unzufriedenheit ob der nicht erledigten Aufgaben gesorgt.

Natürlich sollten Sie auch kompakte Bücher griffbereit haben. Unter anderem ein Buch über Psychopharmakotherapie sowie eine internationale Klassifikation der 
psychischen Störungen (ICD, DSM). In der Kitteltasche hat sich ein kompakter Arzneimittelführer bewährt. So können Sie auch die Ihnen unbekannte Blutdrucktablette in der richtigen Dosierung ansetzen. Alternativ stehen natürlich auch Handy-Apps zur Verfügung.

\section{Kurven und Patientenakten}

Eine wesentliche Informationsquelle auf der Station ist die Patientenkurve und deshalb keinesfalls zu vernachlässigen. Auch wenn die „Kurve“ hoffentlich nicht der einzige Weg ist, der Ihnen einfällt, um mit dem Pflegepersonal zu kommunizieren, so ist es doch ein äußerst wichtiger. Vitalparameter, Blutzuckerwerte und Gewicht sollten auch in der Psychiatrie nicht aus den Augen verloren werden. Allein deshalb verdient die „Kurve“ mindestens einmal täglich einen systematischen Kontrollblick. Unverzichtbar ist dieser Blick auch, um die Medikation des Patienten zu überprüfen: Wurde die Dosierung beim Seitenwechsel richtig übertragen? Haben Sie, wie in der letzten Oberarztvisite besprochen, jene Dosierung im Verlauf der Woche gesteigert und jene gesenkt? Und nimmt die Patientin mit der paranoiden Schizophrenie ihre antipsychotische Medikation regelmäßig ein? Wurde die geplante Blutentnahme oder das Drogenscreening durchgeführt? Die Antwort auf alle diese Fragen verrät Ihnen ein Blick in die „Kurve“. Deshalb sollten Sie ein Ritual daraus machen, zum Beispiel morgens und nachmittags einen Blick in dieses unverzichtbare Instrument $\mathrm{zu}$ werfen. Nebenbei helfen Sie dadurch Ihrem Gedächtnis auf die Sprünge. Sie werden merken: Wenn Sie den Blick in die „Kurve“ ritualisieren, wird es in Zukunft kaum noch vorkommen, dass Sie Ihrem Oberarzt oder der Chefärztin die Antwort auf die Frage nach der aktuellen Medikation eines Patienten schuldig bleiben. Auch werden Sie seltener eine wichtige Kontrolluntersuchung wie eine regelmäßige Lithiumspiegelkontrolle vergessen.

\section{Dem Chaos entgegentreten}

Dies ist vielleicht der wesentliche Punkt für die Arbeit speziell auf einer psychiatrischen Station. Sie werden sich zu Beginn häufig fragen, warum Ihr ausgeklügelter Zeitplan ein ums andere Mal versagt hat und Sie schon wieder Überstunden machen. Oft sind die vielen Störungen gerade in der Psychiatrie ein großer Zeitdieb. Da ist die manische Patientin, die unaufhörlich an Ihrer Tür klopft; da ist der Pfleger, der alle halbe Stunde ruft, weil ein schizophrener Patient ohne Unterlass Fehlhandlungen begeht; da ist der Angehörige, der eine Stunde nach Feierabend ein dringendes Gespräch braucht.

Diesem ständigen Störfeuer kann man nur mit Struktur begegnen: Zunächst gilt, grenzen Sie sich ab! Vergeben Sie Gesprächs- und Visitentermine und halten Sie diese nach Möglichkeit ein - hierbei hilft der eingangs erwähnte Kalender. Machen Sie den Patienten, Angehörigen, Betreuern klar, dass Sie nicht jederzeit zur Verfügung stehen. Nicht nur Sie profitieren davon, sondern auch die Patienten lernen, ihren Tag zu strukturieren. Nicht jeder laut schreiende oder schwer depressive suizidale Patient bedarf der sofortigen Aufmerksamkeit eines Arztes. 
Gerade als Berufseinsteiger fühlen sich viele junge Ärzte für alles verantwortlich und übersehen durch diesen idealistischen hohen Anspruch oft, dass es viele Kollegen anderer Berufsgruppen auf Station gibt, die oft jahrelange Berufserfahrung haben und schwierige Situationen oft besser meistern können. Auch hier gilt jedoch: Ausnahmen bestätigen die Regel. „Störungen“ und „Chaos“ gehören zur Psychiatrie. Nicht immer lässt sich alles planen und manchmal muss ein spontanes entlastendes Einzelgespräch eben doch sein. Indem Sie sich Ihren Tagesablauf strukturieren und lernen, sich abzugrenzen, werden sie aber auch für diese nicht planbaren Situationen mehr Zeit zur Verfügung haben.

\section{Den Arztbriefstapel vermeiden}

Alle Stationsärzte kennen das Gefühl: Der Feierabend nähert sich, gerade ist auf der Station etwas mehr Ruhe eingekehrt und Sie haben das Gefühl, das Tagwerk erfolgreich erledigt zu haben. Plötzlich steht ein Patient vor der Tür und möchte sofort oder spätestens am nächsten Tag entlassen werden. Vielleicht haben Sie durch den hektischen Stationsalltag auch noch die geplante Entlassung eines weiteren Patienten vergessen. Sie schreiben nur einen Kurzbrief mit der Entlassungsmedikation und nehmen sich vor, den ausführlichen Brief am nächsten Tag zu schreiben, was Sie im normal weiterlaufenden Stationsalltag dann vielleicht nicht schaffen. Ganz schnell geraten Sie so ins Hintertreffen und die zu schreibenden Arztbriefe beginnen sich zu stapeln.

Um das zu vermeiden, hilft wieder nur eins: Struktur! Sie sollten es sich zur Routine machen, bei der Neuaufnahme eines Patienten neben dem üblichen Aufnahmeprozedere gleich einen Arztbrief mit anzulegen. Wenn Sie das Glück haben, in Ihrem Haus diktieren zu können, umso besser. Es gibt mehrere Vorteile bei diesem Prozedere: Nach der Aufnahme mit gründlicher Befund- und Anamneseerhebung ist alles noch „frischer“, das Schreiben oder Diktieren geht also leicht von der Hand. Außerdem fällt einem durch dieses Vorgehen häufig frühzeitig auf, was man vielleicht noch nicht erfragt hat oder welche Untersuchung man noch nachholen muss. Zudem sind Sie auf spontane Entlassungswünsche vorbereitet. Sie müssen lediglich noch den Behandlungsverlauf und die Entlassungsmedikation ergänzen und der Brief ist geschrieben. Ein Stapel entsteht erst gar nicht.

\section{Machen Sie sich schlau}

Der vielleicht wichtigste Punkt für einen strukturierten Tagesablauf sind Erfahrung und Wissen. $\mathrm{Zu}$ Beginn ist es oft unvermeidlich, dass Sie länger brauchen als Ihre erfahrenen Kollegen. Angehörige und Patienten stellen Ihnen Fragen, auf die Sie keine Antwort wissen, woraufhin das Vertrauen in Sie als Behandler sinken kann und sich die Therapie zeitaufwendig verkompliziert. Oder vielleicht haben Sie in einem Unterbringungsgutachten ein wichtiges Signalwort vergessen, weshalb Sie es erneut schreiben müssen. Oder Sie müssen sich vor der Eindosierung eines neuen Antidepressivums erst einmal über Nebenwirkungen und Wechselwirkungen schlau machen. Alle diese Dinge kosten Zeit und erklären zu 
einem großen Teil, weshalb Sie als Berufseinsteiger einfach noch nicht so effektiv arbeiten können wie ein erfahrener Kollege. Sie können den Prozess jedoch verkürzen, indem Sie proaktiv und ritualisiert nachlesen. Lesen Sie die Arzneimittelinformation für jedes neu eindosierte Medikament, lesen Sie kurz nach, wenn ein Patient ein Psychotherapieverfahren erwähnt, welches Sie noch nicht kannten oder fragen Sie den Richter, auf welche Formulierungen Juristen Wert legen. Dieses Vorgehen mag am Anfang zeitintensiv sein, auf Dauer ist jedoch fundiertes Wissen der beste Garant für effizientes Arbeiten, ganz nebenbei auch für Ihre eigene Zufriedenheit. Kurzes zeitnahes Nachlesen oder Nachfragen ist oft sehr effektiv, da es sich nicht um rein theoretisches Lernen handelt, sondern der unmittelbare Praxisbezug die Relevanz unterstreicht. Sie haben den konkreten Patienten vor Augen, ihr Wissensgewinn hat einen unmittelbaren Effekt.

\section{Vernachlässigen Sie die Somatik nicht}

Ihr Patient hat Fieber und eine Tachykardie und alles was Ihnen einfällt ist, den Konsilarzt der Inneren Medizin zu rufen? Bei einer Patientin fällt dem Pflegepersonal eine Parese der linken Körperhälfte auf und Sie können nicht sagen, ob diese schon bei Aufnahme bestand, weil Sie nicht gründlich neurologisch untersucht haben? Eine Krankenpflegerin ruft Sie in der Nacht an und fragt, was sie bei der Unterzuckerung einer Patientin mit Typ-I-Diabetes tun soll und Sie haben keine Ahnung? Das sind die Momente, in denen Sie sich nicht nur schnell den Ruf erarbeiten können, „kein richtiger Arzt“ zu sein, sondern im schlimmsten Fall auch Ihre Patienten gefährden können. Zudem kostet Unwissen hier extrem viel Zeit. Deshalb nochmal zum Mitschreiben: Sie sind Arzt! Um stressfrei in den Beruf zu starten, bereiten Sie sich also bitte auch auf die "Somatik“ vor. Absolvieren Sie Ihre Famulaturen nicht nur in der Psychiatrie. Zeigen Sie auch im chirurgischen und internistischen Tertial des Praktischen Jahres Engagement. Und bilden Sie sich auch im somatischen Bereich fort. Wenn Sie beispielsweise einen alkoholabhängigen Patienten mit Pankreatitis konsiliarisch Ihrem internistischen Kollegen vorstellen wollen, lesen Sie sich kurz in das entsprechende Krankheitsbild, Diagnostik und Therapie ein. So können Sie die Konsilanforderung prägnant formulieren und notwendige Untersuchungen bereits einleiten. Es kommt auch nicht gut an, wenn Sie einen Patienten mit unklarem Fieber über $38,5^{\circ} \mathrm{C}$ konsiliarisch vorstellen, ohne den Patienten gründlich untersucht $\mathrm{zu}$ haben. Auch Sie können eine Fokussuche beginnen, eine Urinuntersuchung veranlassen, die Lunge auskultieren, eine Blutentnahme durchführen und eine Flexüle legen, um eine Flüssigkeitssubstitution zu beginnen. Sie müssen nicht alles wissen und Sie dürfen alles fragen, aber informieren Sie sich, bevor Sie einen fachärztlichen Kollegen unter Zeitdruck rufen. Schließlich wollen Sie auch nicht von einem internistischen Kollegen gerufen werden, um „mal mit dem Patienten zu reden“ bevor der Kollege selbst dies überhaupt versucht hat. Wenn die Kollegen merken, dass Sie sich Ihre Gedanken machen, werden sie Sie ernst nehmen. Dann können Sie Ihre Fragen stellen und von der interdisziplinären Zusammenarbeit profitieren. 


\section{Tipps für eine bessere Organisation des Tages}

- Setzen Sie Prioritäten.

- Benutzen Sie Hilfsmittel.

- Werfen Sie regelmäßig einen Blick in Patientenkurven und -akten.

- Vergeben Sie Termine.

- Legen Sie Arztbriefe gleich nach der Patientenaufnahme an.

- Vertiefen Sie Ihr Wissen durch zeitnahes Nachlesen und Nachfragen.

- Bleiben Sie auch somatisch auf dem Laufenden.

\section{Teamarbeit}

Ohne das Team läuft auf einer psychiatrischen Station gar nichts. Damit Sie von der „Ressource Team“ profitieren können, beherzigen Sie die folgenden Ratschläge.

\section{Sich ins Team einfügen}

Beim Berufseinstieg ist alles neu und aufregend. Die Patienten müssen versorgt werden. Wissenslücken wollen gestopft werden. Die Vorgesetzten sollen einen guten Eindruck vom neuen Mitarbeiter bekommen.

Da wird häufig vergessen, dass der erste Eindruck, den die Mitarbeiter auf der Station von einem bekommen, für den späteren Alltag mindestens genauso wichtig ist. In den ersten Wochen entscheidet sich, ob man es Ihnen auf Station leicht oder schwer macht, Teil des Teams zu werden. Ein freundlicher Umgangston und Hilfsbereitschaft haben noch keinem Berufseinsteiger geschadet. In den ersten Tagen sollten Sie sich jedem Mitarbeiter vorstellen, ganz gleich, ob Reinigungskraft, Fahrer, Pflegeschüler, Physiotherapeut oder Hausmeister. Sie sollten sich in den ersten Wochen nicht in Ihrem Zimmer verkriechen, sondern mit offenen Augen über die Station laufen. Lernen Sie die Mitarbeiter und ihre Funktionen und Tätigkeiten kennen, hospitieren Sie in der Ergotherapie, schauen Sie sich psychotherapeutische Gruppensitzungen an, erkundigen Sie sich beim Pflegepersonal, welche weiteren Angebote es auf der Station gibt. Für welchen Patienten eignen sich welche Therapieprogramme, wer darf wann die Station verlassen, wo findet eigentlich die Physiotherapie statt? Die Antworten auf diese Fragen wissen erfahrene Mitarbeiter besser als jedes Buch. Trauen Sie sich zu fragen. Den Kollegen sollte klar sein, dass Sie Berufseinsteiger sind und nicht alles wissen können.

\section{Sich in die Lage anderer Berufsgruppen versetzen}

Spätestens nach Ihrem ersten Nachtdienst werden Sie der Überzeugung sein, dass Sie es als Arzt am allerschwersten haben. Selbstmitleid führt aber leider häufig zu einem Tunnelblick. Seltsamerweise sind viele in der Psychiatrie tätige Mitarbeiter im Kontakt mit den Kollegen anderer Berufsgruppen nicht so empathisch, wie sie es im Kontakt mit den Patienten sind. Deshalb hilft es, sich immer 
wieder in Erinnerung zu rufen, dass es trotz der hohen Verantwortung, beispielsweise eines Dienstarztes, meistens die Pflegekräfte sind, die die anstrengendsten Situationen zu meistern haben. Sie sind den Konflikten auf Station oft unmittelbar ausgesetzt, häufig, ohne sich wirklich zurückziehen zu können. Und wenn ein Patient Sie durch sein ständiges Klopfen an Ihrem Dienstzimmer in den Wahnsinn treibt, so können Sie sicher sein, dass er es bereits um ein Vielfaches häufiger beim Pflegepersonal probiert hat. Auch bei Notfallsituationen, wie Suizidversuchen oder stark fremdaggressivem Verhalten, sind meistens die Pflegekräfte diejenigen, die am nächsten dran sind. Deshalb hilft es bei Konflikten auf Station, einen Perspektivwechsel zu versuchen. Warum ist die Atmosphäre auf der Station seit einer Woche so angespannt? Warum hat jener Pfleger ein so großes Problem mit besagtem Patienten? Warum wird in jeder Pflegeübergabe in gereiztem Tonfall auf Sie eingeredet, damit Sie die Ihrer Ansicht nach freundliche, gut mitarbeitende Patientin entlassen? Besprechen Sie dies im Team und betrachten die Lage nicht nur aus der Arztperspektive. Nehmen Sie von Zeit zu Zeit an der Pflegeübergabe teil und sorgen Sie dafür, dass in Teamsitzungen jede Berufsgruppe zu Wort kommt.

\section{Die Meinung der Kollegen ernst nehmen}

Das Schöne an einem Stationsteam ist, dass unterschiedliche Kollegen mit unterschiedlichen Erfahrungshorizonten und Ausbildungen gemeinsam in der Regel bessere Entscheidungen treffen. Davon sollten Sie profitieren. Deshalb gilt als wichtigste Regel: Legen Sie Ihren möglicherweise vorhandenen „Arztdünkel“ ab. Machen Sie sich klar: Assistenzärzte kommen und gehen. Krankenpfleger sind hingegen oft seit Jahren oder Jahrzehnten in ihrem Bereich tätig und somit echte Spezialisten, deren Meinung unverzichtbar ist. Hinzu kommt, dass sie oft bessere Informationen über die Patienten haben, diese vielleicht bereits von früheren Aufenthalten kennen oder aber im Stationsalltag ganz anders erleben als der Arzt in seiner kurzen Visite. Selbiges gilt für andere Berufsgruppen wie Psychologen, andere Therapeuten und Sozialarbeiter. Sie müssen und sollten Entscheidungen also nicht allein treffen, sondern sie im Team besprechen. Eine offene Diskussion und eine transparente Entscheidungsfindung sind wesentlich. Denn nur, weil unter vielen Dokumenten eine Arztunterschrift stehen muss, heißt das nicht, dass Sie alleiniger Entscheider sind. In diesem Sinne sollten auch schwierige Situationen auf Station, wie beispielsweise eine belastende Fixierungssituation, im gesamten Team nachbesprochen werden und konstruktiv nach Verbesserungen gesucht werden.

\section{Nicht alles allein machen}

Machen Sie sich klar, wer auf Station welche Aufgabe hat. Gibt es einen Stationsassistenten, der das Fax an das Amtsgericht vielleicht in der Hälfte der Zeit schickt, weil er das mehrmals pro Woche macht und die Nummern parat hat? Müssen immer Sie es sein, der ans Telefon gerufen wird, wenn ein ehemaliger Patient einen Brief aus dem Archiv haben will? Und warum kann die gesetzliche 
Betreuerin eines anderen Patienten nicht auch mal mit dem Sozialdienst der Station über einen möglichen Wechsel der Wohneinrichtung sprechen? All dies sind Beispiele von alltäglichen Situationen, die enorm viel Zeit rauben können und leicht von anderen Kollegen übernommen werden können. Hierbei gilt es, nach und nach herauszufinden, wie die Aufgaben auf der Station verteilt sind und anschließend freundlich, aber bestimmt zu delegieren. Das heißt nicht, dass Sie wie ein Halbgott in Weiß über die Station rauschen und es sich mit allen Kollegen verscherzen. Ja, auch Sie können mal ein Fax schicken, wenn der Stationsassistent gerade im Stress ist. Wenn Sie jedoch allein unzählige Überstunden machen und den halben Tag mit Korrespondenz statt mit Diagnostik und Therapie beschäftigt sind, läuft etwas falsch.

\section{Sachlich bleiben}

Patientenverhalten kann ein Team durch zum Beispiel emotional instabile oder stark provozierende Handlungen spalten. Dadurch, dass die Emotionen bei vielen Patienten so unkontrolliert hervortreten, löst dies unvermeidbar auch bei den Mitarbeitern bestimmte emotionale Verhaltensweisen aus. So kann es zum Beispiel passieren, dass Ihnen ein Mitarbeiter Vorwürfe macht, weil Sie einen sich anzüglich verhaltenden Patienten nicht entlassen, obwohl dieser Ihrer Meinung nach schwer suizidal ist. Sie werden sicherlich sofort zustimmen, dass die folgende Replik indiskutabel wäre: „OK, dann entlassen wir ihn halt. Aber wenn er sich umbringt, sind Sie schuld!“ Dennoch werden Sie solche Sätze im Stationsalltag zu hören bekommen. Wir sind alle Menschen und haben unsere Emotionen nur mehr oder weniger im Griff. Deshalb ist es umso wichtiger, in Diskussionen im Team sachlich zu bleiben und das Für und Wider abzuwägen. Die Patienten sind schließlich bereits emotional genug. Zum Beispiel kann man im Team gewisse Störungsbilder sachlich erklären oder diskutieren, warum jener Patient anzügliche Bemerkungen macht.

\section{Umgang mit Hierarchien}

Machen Sie sich nichts vor. Trotz vieler anderslautender Beteuerungen ist das System Krankenhaus weiterhin ein hierarchisches System, wenn nicht eins der am stärksten hierarchisch geprägten Systeme in der modernen Arbeitswelt. Die Psychiatrie macht da trotz ihres Rufs, ein „weiches Fach“ zu sein, keine Ausnahme. Psychiater mögen Patienten noch so oft vermitteln, wie sie eine gesunde Work-Life-Balance erzielen, Arbeitskonflikte lösen oder ihre Meinung vertreten sollen. Sobald sie ein Krankenhaus oder eine Abteilung leiten, scheinen diese Grundsätze zumindest für die eigenen Mitarbeiter oft nicht mehr zu gelten. Auf der anderen Seite sind auch Assistenzärzte eine, was ihre eigenen Belange angeht, eher passive Berufsgruppe. Dies mag auch an befristeten Arbeitsverträgen liegen und der Tatsache, dass man sich ja schließlich noch in der Ausbildung befindet und Lehrjahre bekanntlich keine Herrenjahre sind. Nur sind die Lehrjahre bei jungen Medizinern weitaus länger als bei vielen anderen Berufen. Machen Sie sich klar, welche Expertise und Lebenserfahrung Sie in die Waagschale 
werfen und vertreten Sie Ihre Interessen und Meinungen, wenn es darauf ankommt. Denn ein Beruf ist nur dann wirklich erfüllend, wenn man das Gefühl hat, nicht nur Erfüllungsgehilfe zu sein, sondern eigene Werte und Ideen mit einbringen zu können, oder zumindest nicht dagegen verstoßen zu müssen. Es gilt, einen gesunden Mittelweg zu finden. Zunächst sollten Sie versuchen, das System und die hierarchische Ordnung zu verstehen, um nach und nach mehr von sich einbringen $\mathrm{zu}$ können. In welcher Mitarbeiterrunde ist Ihre Meinung gefragt, wann sollten Sie besser schweigen? Welche Zusatzaufgabe wird auch von Ihren Kollegen als Selbstverständlichkeit angesehen und was ist vielleicht eine Überbelastung? Wird Ihr Oberarzt sich freuen, wenn Sie Änderungsvorschläge oder Neuerungen einbringen, oder wird dies als Anmaßung empfunden? Im Allgemeinen sollten Sie zu Beginn beobachten, sich zurückhalten, von Kollegen lernen. Mit wachsender Berufserfahrung sollten Sie Ihr Licht aber auch nicht unter den Scheffel stellen, sondern sich aktiv einbringen und auch Ihre eigenen Interessen vertreten.

\section{Genug geredet!}

Falls Sie aus einer anderen Fachrichtung in die Psychiatrie wechseln, wird es Ihnen vielleicht besonders auffallen: In Psychiatrie-Teams wird viel geredet. Es gibt unzählige Übergaben, Teamsitzungen, Supervisionen, Fortbildungen und Besprechungen. Das ist einerseits eine große Stärke der Psychiatrie. Allerdings kann es auch ganz schnell zu einem Nachteil werden, wenn diese Sitzungen zum Selbstzweck werden, wenn Dinge eher „zerredet“ werden als zu einem Ziel zu führen. Dann führt Teamarbeit nicht zu dem eigentlichen Ziel der verbesserten Patientenversorgung, sondern zu Ineffektivität und Unzufriedenheit. Wenn Sie an einem hektischen Tag das Gefühl haben, dass die Teambesprechung gerade nicht besonders fokussiert und zielführend läuft, versuchen Sie Fokus und Struktur wiederherzustellen. Falls das nicht funktioniert, weisen Sie freundlich und bestimmt auf den Zeitplan hin. In der Regel sind die anderen Mitarbeiter dafür dankbar. Schließlich hat jeder sein Tagwerk zu erledigen.

\section{Tipps für die Teamarbeit}

- Der erste Eindruck ist wichtig: Zeigen Sie sich offen und hilfsbereit.

- Versuchen Sie, die Perspektive anderer Berufsgruppen zu verstehen.

- Profitieren Sie vom Wissen und der Erfahrung anderer Mitarbeiter.

- Lernen Sie, gewisse Aufgaben zu delegieren.

- Bleiben Sie bei Konflikten sachlich.

- Finden Sie Ihren Platz im hierarchischen System.

- Achten Sie bei Teamarbeit auf Fokus und Zielführung. 